\title{
ACTITUDES HACIA LA CIENCIA Y ASIGNATURAS PENDIENTES: DOS FACTORES QUE AFECTAN AL RENDIMIENTO EN CIENCIAS ${ }^{(*)}$
}

\author{
ESPINOSA, J., ROMÁN, T. \\ Seminario Permanente de Física y Química: «Vegas Altas del Guadiana» \\ I.B. «Luis Chamizo», 06400 Don Benito (Badajoz).
}

(*) En memoria del Dr. J.L. Mesías Iglesias.

\section{SUMMARY}

This paper analyses the influence of the attitude towards Science with which some students attain secondary education (BUP) and the role of outstanding subjects in their school record.

\section{INTRODUCCIÓN}

El rendimiento escolar es un problema multifactorial que ha sido ampliamente analizado desde diversos puntos de vista. Desde factores socioeconómicos (González 1982), amplitud de programas, metodología o masificación, pasando por los preconceptos científicos o conceptos alternativos con los que los alumnos llegan a BUP (Viennot 1977, Caamaño 1983, Furío 1983, Gil 1983, García 1985, Sebastiá 1985, Driver y col. 1985, Clough y Driver 1986, Novak 1987, Carrascosa 1987, Hierrezuelo y Montero 1989) hasta el nivel de pensamiento formal, pues algunas materias que se imparten en BUP y COU requieren un nivel de pensamiento formal que muchos alumnos aún no han alcanzado (Piaget 1972, Aguirre 1983, Barrios 1985).

Por nuestra parte, quisiéramos llamar la atención sobre dos aspectos que suelen pasar desapercibidos en la investigación didáctica española y que pueden representar un peso estadístico importante en el fracaso escolar. Nos referimos concretamente a la actitud de los alumnos hacia la Ciencia y a la poblemática de las asignatıras "pendientes", que a nuestro juicio ha sido infravalorada en la investigación didáctica. Pensamos que se tratá de temas de crucial importancia cuantitativa (como después veremos), que podrían incluso condicionar planteamientos didácticos y metodológicos.

\section{ACTITUD HACIA LA CIENCIA}

\section{A) Hipótesis de trabajo y diseño de la experiencia}

En un reciente trabajo (Yager y Penick 1986) se constata que «cuantos más años de Ciencias cursan nuestros alumnos menos les gusta», debiéndose este descenso progresivo de intereses escolares fundamentalmente a dos tipos de factores (Yager y Penick 1986, Solbes 1989):

a) Factores externos al aula, como son los socioeconómicos, familiares, etc., de difícil actuacion por parte del profesor.

b) Factores internos, como falta de interés en actividades escolares, reducidas a memorización y repetición de libros de texto, tipo de evaluación, papel del profesor, dinámica del centro, imagen deformada de Ciencia y los científicos, etc., sobre los cuales puede incidir el profesor.

Por otra parte, se constata (Serrano 1988) que mientras que a los alumnos de $5^{\circ}$ de EGB les resulta más ameno estudiar Ciencias Experimentales que Ciencias Sociales - Matemáticas, al terminar la escolaridad obligatoria ha variado su percepción de la amenidad del estudio de las Ciencias: les parecen más aburridas y desagradables que en $5^{\circ} \mathrm{de} \mathrm{EGB}$. 
Estc cambio en las actitudes de los alumnos ha sido investigado por un gran número de autores, entre los que podemos destacar a Giordan (1982), Klopfer (1976), Mager (1985), Hadden y col. (1983), Schibeci (1986) y Serrano (1986).

Por nuestra parte, hemos pretendido profundizar en el conocimiento de la actitud respecto a la Ciencia con la que los alumnos llegan al BUP desde la $2^{2}$ etapa de la EGB.

Entre los instrumentos publicados para conocer la actitud de los alumnos (Summers 1976, Fraser 1978, Krajkovich 1982, Chambers 1983 y Ridgeng 1984) hemos elegido la elaboración de una encuesta (Fig. 1) basada en las ideas de M. Belmonte (1979) donde se plantean tana serie de cuestiones contrarias que el alumno debe puntuar de 0 a 10 , colocando a la izquierda las actitudes que a nucstro criterio son positivas y a la derecha las negativas, siempre dentro de unos márgenes de subjetividad entre las ideas positivo/negativo.

La encuesta fue realizada a 393 alumnos de $1^{\prime \prime}$ de BUP de cinco centros de la zona durante el primer mes del curso $88-89$.

\section{B) Resultados y discusión}

Los resultados de la encuesta, (Fig. 1), aparecen reflejados en la tabla I. La experiencia acumulada por estos alumnos durante la segunda etapa de ta FGB les hace difícil y liosa la Física y Química (cuestiones 2,3 y 6 ), aunque la cncuentran interesante (cuestiones 1,4 y 7 ). Es de destacar la cuestión 10, que alcanza el valor medio más alto, donde los alumnos indican que valían la pena las dificultades encontradas en esta etapa.

\section{Figura 1}

En los cursos de $7^{\text {n }}$ y $8^{\prime \prime}$ de FGB estudiaste la Física y La Química. A continuación se te indican una serie de adjetivos $n$ situaciones contrarias. Señala con una puntuación entre 0 y 10 cada una de ellas, de modo que si crees que la cuestion se identifica con lo expuesto a la izquierda marca el 10, si con el de la derecha marca el 0 , si es una situación intermectia, según su proximidad a uno u otro adjetivo, ucercate más o menos en la puntuación à uno de los extremos.
1. INTERESANTE
109876543210
2. CL.ARO
3. F̈̈CIt.
4. ME HA ACERCADO
A LA NATURALEZA
5. PRORARIA OTRA VEZ
109876543210
ABURRIDO
109876543210
LIOSO
109876543210
DIFICIL
6. ME HA COSTADO
109876543210
7. HE: APRENDIDO,
FISICA Y QLÍMICA
8. ME HA GUSTADO
9. ME HE DJVERTIDO
109876543210
109876543210
109876543210
10. VAIÍA LA PENA

labla I

\begin{tabular}{|c|c|c|}
\hline CUI:STIÓN & MEDIA & $\begin{array}{l}\text { DESVIACION } \\
\text { DE LA MTDDIA }\end{array}$ \\
\hline $\begin{array}{l}\text { 1. INTFRFSANTE/ABLRRIDO) } \\
\text { 2. CLARO/LIOSO } \\
\text { 3. FACIL/DIFICIL } \\
\text { 4. MHE HA ACERCADO A } \\
\text { LA NATURALEZA/ } \\
\text { NI YNN BROMA } \\
\text { 5. PROBARIA OTRA VEZ/JAMAS }\end{array}$ & $\begin{array}{l}6,3 \\
6,3 \\
6,0\end{array}$ & $\begin{array}{l}2,8 \\
2,9 \\
2,8\end{array}$ \\
\hline $\begin{array}{l}\text { 6. ME HA COSTADO/FACIL } \\
\text { 7. HE APRENDIDONNADA } \\
\text { 8. ME HA GUSTADONNADA } \\
\text { 9. ME JIE DIVERTIDO/ } \\
\text { IE SLFRIDO } \\
\text { 10. VALIA I.A PENA/ } \\
\text { TIEMPO PERDIDO }\end{array}$ & $\begin{array}{l}6,3 \\
6,0 \\
5,7 \\
5,3 \\
7,4\end{array}$ & $\begin{array}{l}2,6 \\
2,5 \\
3,0 \\
2,7 \\
2,5\end{array}$ \\
\hline
\end{tabular}

Por otra parte, estábamos interesados en cuantificar de alguna manera estos resultados, por lo que construímos una escala arbitraria de puntuaciones. Así, hemos agrupado en cada pregunta el número de alumnos con puntuaciones 0-3 (actitud negativa), 4-6 (indiferente) y 7-10 (actitud positiva), obteniendo los resultados de la tabla II.

Con estos datos, nos encontramos que un $19,3 \%$ de alumnos presenta una actitud negativa hacia la Física y Química en particular y la Ciencia en general; un $34,7 \%$ una actitud indiferente y un $46,0 \%$, una actitud positiva. Estos resultados son reveladores de la actitud de los alumnos cuando se enfrenten a la Física y Química el curso próximo ( $2^{\circ}$ de $B(i P)$ y, de alguna manera, podrían explicar el elevado fracaso escolar en nuestra asignatura.

Tabla II

\begin{tabular}{rrrrrrrrrrrrr} 
Calificación & 1 & 2 & 3 & 4 & 5 & 6 & 7 & 8 & 9 & 10 & Toral \\
\hline 0.3 & 65 & 73 & 71 & 105 & 112 & 55 & 55 & 88 & 102 & 31 & 757 \\
4.6 & 123 & 120 & 140 & 146 & 119 & 153 & 164 & 134 & 171 & 92 & 1362 \\
7.10 & 205 & 200 & 182 & 142 & 162 & 185 & 174 & 171 & 120 & 270 & 1811 \\
\hline$\ldots$ & & & & & & & & & & & &
\end{tabular}

Las razones de esta actitud negativa son multifactoriales, pero nuestra experiencia acumulada durante años impartiendo cursos de perfeccionamiento a profesores de FGB, nos indica que una de ellas puede ser la no correspondencia entre los contenidos que se imparten en el aula y la capacidad de razonamiento de los alumnos; así como la falta de coordinación entre los niveles educativos. En este sentido quisiéramos recordar las ideas de un viejo profesor de universidad cuando decía que era preferible que no explicásemos determinados contenidos difíciles a que el alumno llegara a odiar una asignatura por encontrarla muy árida. En el primer caso, siempre puede llegar otro profesor en cursos venideros y cxplicárselo, en el segundo caso esto sería imposible. 
Tabia III

\begin{tabular}{lccccccc} 
Calificaciones & $\operatorname{Sob}(9)$ & $\operatorname{Not}(7)$ & $\operatorname{Bien}(6)$ & $\operatorname{Suf}(5)$ & $\operatorname{Ins}(4)$ & $\mathrm{MD}(2)$ & Total Alumn. \\
\hline «CON PENDIENTES» & 0,8 & 2,5 & 4,2 & 17,6 & 30,2 & 44,7 & 119 \\
«SIN PENDIENTES» & 7,3 & 14,6 & 16,0 & 32,3 & 15,6 & 14,2 & 275
\end{tabular}

\section{INFLUENCIA DE LAS ASIGNATURAS PENDIENTES}

\section{A) Hipótesis de trabajo y diseño de la experiencía}

$\mathrm{Si}$ el punto anterior ha recibido una amplia y profunda atención por parte de numerosos autores, no podemos decir lo mismo en cuanto a este segundo punto. Las asignaturas pendientes ejercen una gran influencia sobre el rendimiento escolar de los alumnos. Esta influencia no ha sido suficientemente tratada y, a nuestro juicio, ha pasado desapercibida en la investigación didáctica, aunque Arana y col. (1987) han estudiado la influencia que ejerce el hecho de repetir curso sobre las actitudes de los alumnos con respecto a las Ciencias.

En este segundo punto intentamos considerar la existencia de las asignaturas pendientes como un índice de fracaso escolar en general y, más concretamente, como una causa de bajo rendimiento en la asignatura de Física y Química de $2^{\circ}$ de BUP.

El estudio se ha realizado sobre un total de 394 alumnos de $2^{\circ}$ de BUP matriculados en tres centros de la zona, una vez finalizado el curso 86-87, teniendo en cuenta las calificaciones en la asignatura de Física y Química y eI número de asignaturas suspensas en Junio, para las pobiaciones de «alumnos con pendientes» (Grupo A) y «alumnos sin pendientes» (Grupo B).

\section{B) Resultados y discusión}

En la tabla III aparecen los porcentajes de alumnos que han obtenido las distintas calificaciones de Física y Química en la convocatoria de Junio, referidos a las dos poblaciones estudiadas (con pendientes y sin pendientes).

De los 119 alumnos del grupo A, el $75 \%$ no aprueba la Física y Química de $2^{\circ}$ de BUP en Junio, quizá porque su nivel de pensamiento formal no está relacionado con los contenidos que se imparten y/o presentan deficiencias en el cálculo matemático necesario para esta asignatura (Fernández 1986).

Por otra parte, el $52 \%$ de los alumnos que suspenden esta asignatura en Junio corresponde a alumnos del grupo $A$. Quisiéramos llamar la atención sobre el tradicional «fracasoy en esta asignatura y relacionarlo, al menos en parte, con la influencia del factor estudiado.

Aparte del estudio puntual de la influencia de las asignaturas «pendientes» en nuestra asignatura, también hemos analizado el efecto del factor estudiado sobre la marcha general del curso. En la tabla IV aparece el número de alumnos frente al número de asignaturas suspensas de Junio, para las dos poblaciones estudiadas.

En primer lugar, de los alumnos «sin pendientes» aprueban todas las asignaturas el $50 \%$ frente al $5 \%$ del grupo "con pendientes». Además, si consideramos como rendimiento aceptable de un curso hasta un máximo de dos asignaturas suspensas en Iunio (no olvidemos que la edad media de los alumnos está sobre los 14-15 años, donde los problemas más emotivos y de madurez son importantes), el $77 \%$ de los alumnos del grupo B pueden considerarse en este apartado, frente a tan sólo el $24 \%$ del grupo A. O de otra manera, solamente el $24 \%$ de los alumnos con asignaturas "pendientes》 del $1^{2}$ de BUP aprovecha satisfactoriamente el curso siguiente (la palabra satisfactoria corresponde al límite que hemos establecido de dos asignaturas suspensas en Junio, que como es obvio, es totalmente arbitrario).

En resumen, de todo lo dicho podemos deducir que los dos aspectos comentados en este trabajo representan un problema cuantitativo serio. Las diferentes líneas de investigación didáctica y metodológica deberían contemplar esta problemática para una mejora de la calidad del proceso enseñanza/aprendizaje de las Ciencias en general y de la Física y Qúmica en particular.

Tabla IV

\begin{tabular}{lrrrrrrrrrrr} 
Número de Asignaturas & \multicolumn{11}{c}{ Total } \\
\hline Suspendidas en Junio & 0 & 1 & 2 & 3 & 4 & 5 & 6 & 7 & $\times$ & Sx & Alumn. \\
«CON PENDIENTES» & 6 & 9 & 14 & 18 & 14 & 17 & 21 & 20 & 4,2 & 2,1 & 119 \\
«SIN PENDIENTES» & 135 & 47 & 30 & 20 & 19 & 14 & 8 & 2 & 1,4 & 1,8 & 275 \\
\hline
\end{tabular}




\section{REFERENCIAS BIBLIOGRÁFICAS}

AGUIRRE DE CARCER, J., 1983. Dificultades de la comprensión de la explicación en los libros de texto de Física, Enseñanza de las Ciencias, Vol. 1(2), pp. 92-98.

ARANA, I., ESCUDFRO, T., GARCÉS, R, y PALACÍN, F, 1987, Enseñanza de las Ciencias, Vol, 5 (1), pp. 10-15.

BARRIOS, M., 1985. Relationships between cognitive development and Science-related attitudes on Science achievement, Enseñanza de las Ciencias, Vol. extra, p. 19.

BELMONTE, M., 1979. El método activo para el descubrimiento personalizado de las ciencias como alternativa a su cnseñanza tradicional: realización práctica en Ia Física y Química de $2^{\circ}$ y $3^{\circ}$ de Bachillerato, Revista de Bachillerato, 10 , pp. 71-84.

CAAMAÑO, A. et al, 1983. Consideraciones sobre algunos errores conceptuales en el aprendizaje de la Química en el Bachillerato, Enseñanza de las Ciencias, Vol. 1(3), pp. 198200.

CARRASCOSA, J., 1987. Tratamiento didáctico en la enseñanza de las Ciencias de los errores conceptuales. I'esis Doctoral. Universidad de Valencia.

CHAMBERS, D, 1983. Stcrcotypic images of the scientists: the Draw a Scientist test, Sci. Education, 67(2), pp. 255-265.

CLOUGH, E.E. y DRIVER, R., 1986. A Study of consistency in the use of students'conceptual framework across different task context, Sci. Education, 70 (4), pp. 473-496.

DRIVER, R.,GUESNE, E. y TIBERGHIEN, A., 1985. Children's Ideas in Science. Open University Press.

FERNÁNDEŹ FERNÁNIDEZ, I.M. et a1., I986. Recursos matemáticos de los alumnos que comienzan los estudios de Física y Química en BUP, Aula Abierta, 46, pp. 143-151.

FRASER, B., 1978. Development of a test of Science related attitudes (TORSA), Sci. Education, 62(4), pp. 509-515.

FURIO MAS, C. y ORTTZ, E., 1983. Persistencia de errores conceptuales en el equilibrio químico, Enseñanza de las Ciencias Vol. 1(1), pp. 15-20.

GARCÍA-HOURCADE, J.L. y RODRIGUEZ DE ÁVILA, C., 1985. Preconcepciones sobre el calor en $2^{\circ}$ de BUP, Enseñanza de las Ciencias, Vol.3(3), pp. 188-193.

GIL PEREZ, D., 1983. Tres paradigmas básicos en la enseñanza de las Ciencias, Enseñanza de las Ciencias, Vol. 1(1), pp. 26-33.

GIORDAN, A.,1982. Liberación. Prioridad de la actitud científica, Enseñanza de las Ciencias, Pablo del Río, S. XXI, Madrid, pp. 57-111.

GIORDAN, A., 1985. Interês didáctico de los errores de los alumnos, Enseñanza de las Ciencias, Vol. 3(1), pp. 11-17.

GONZÁLEZ ESTÉVEZ, M.A., 1982. Incidencia del entomo socioeconómico sobre la marcha académica del alumno, Revista de Bachillerato, 23, pp. 68-73.
HADDEN, R. y JOHNSTON, A., 1982. Primary school pupils'attitudes to science: The years of formation, Eur. $J$. Sci. Education, 4(1), pp. $397-407$.

HADDEN, R. y JOHNSTON, A., 1983. Secondary school pupils'attitudes to science: 'The year of erosion, Eur. $J$. Sci. Education, 5(3), pp. 309-318.

HADDEN, R, y JOHNSTON, A., 1983. Secondary school pupils attitudes to science: The year of decision, Eur.J.S S $i$. Education, 5(4), pp. 429-438.

HIERREZLEI,O, J. y MONTERO, A., 1989. La Ciencia de los alumnos. (Laia-MEC).

KLOPFER, L., 1976. A structure for the affective domain in relation to science education, Sci, Educ. 60(3), pp. 299-312,

KRAJKOVICH, F. y SMITH, J., 1982. The development of image of science and scientist scale, J.R. Sci. Teach, 19(1), pp. 9-44.

MAGER, R., 1985. Desarrollo de actitudes ante la enseñanza. (Martínez Roca: Barcelona).

NOVAK, J., 1987. Proceding Seminar. Misconceptions and Educational strategies in Science and Mathematics. Cornell.

PIAGET, J., 1972. Intelectual evolution from adolescence to adulthod, Human Development, Vol. 15, pp. 112.

RIDGENG, J. y SCHIBECI, R, 1984. The development and validation of a test of biology related attitudes, Res. Sci. and Teach. Education, 12(1), pp. 2I-29.

SEBASTI $\dot{A}, J . M ., 1985$. Interpretaciones espontáneas de la dinámica del movimiento, Enseñanza de las Ciencias, Vol. extra, pp. 63

SCHIBECI, R.A., 1982. Measuring student attitudes: semantic differential or likert instruments, $S c i$. Educatiom, $66(44)$, pp. 565-570.

SCHIBECI, R.A., 1986. Images of science and scientists and science education, Sci. Education, 70(2), pp. 139-150.

SERRANO, T., I986. La imagen de los científicos en los alumnos al finalizar el ciclo medio. Documentos IEPS, monografía $n^{\mathrm{a}} 1$.

SERRANO, T., 1988. Actitudes de los alumnos y aprendizajo de las ciencias. Ln estudio longitadinal, Investig. Escuela, 5 , pp. 29-38.

SOLBES, J., 1989. Segundo curso de Formación de Formadores en Didáctica de las Ciencias. Valencia.

SUMMERS, G., 1976. Medición de actitudes. (Trillas: Méjico).

VIENNOT, L., 1977. Le raisonnement spontané des élèves en dynamique. Tesis doctoral. Lniversidad de Paris 7.

YAGER, R.F. y PENICK, J.E., 1986. Perceptions of four age groups towards science classes, teachers and the value of science, Sci. Education, 70(4), pp. 355-364. 